\title{
Social Support for Chamorro Breast Cancer Survivors on Guam
}

\author{
Lilli Ann Perez ${ }^{1}$, Lisalinda Natividad ${ }^{l}$, William Chung ${ }^{2}$, Robert L. Haddock ${ }^{3}$, \\ Lari Wenzel ${ }^{2,4}$ and F. Allan Hubbell ${ }^{2,4}$ \\ ${ }^{1}$ Family Pacific \\ ${ }^{2}$ Health Policy Research Institute \\ ${ }^{3}$ Cancer Research Center of Guam, University of Guam \\ ${ }^{4}$ University of California, Irvine, Department of Medicine and \\ Chao Family Comprehensive Cancer Center
}

\begin{abstract}
Purpose: The purpose of this study was to assess the types of social support used by Chamorro (indigenous) breast cancer survivors on Guam.

Methods: We assessed social support use among 25 self-reported Chamorro women with a diagnosis of breast cancer through interviews and construction of genograms and ecomaps -pictorial displays of the women's family relationships, medical history, and their social networks.

Results: The mean age of the participants was 54.5 years. The average number of years since the diagnosis of breast cancer was 7.8 years. Respondents indicated that the nuclear family was the most important form of social support (34.2\%). Indeed, nuclear family and other types of informal systems were the most common type of social support used by the women $(60.2 \%)$. Formal support services, clubs, and organizations were reported by $17.9 \%$ of participants while spiritual and/or religious resources were reported by $21.9 \%$ of them.

Conclusion: These Chamorro breast cancer survivors depended largely on family for social support. Support from family, although informal, should be recognized as a pivotal factor in recovery and survivorship. Future directions could incorporate formal and informal mechanisms to utilize this natural support resource.
\end{abstract}

(c) 2010 Californian Journal of Health Promotion. All rights reserved.

Keywords: breast cancer, survivorship, social support

\section{INTRODUCTION}

Breast cancer is the most commonly diagnosed cancer and the leading cause of death due to cancer among Chamorro (indigenous) women on Guam (Haddock, 2002 \& Haddock, 2006). The age-adjusted incidence rate $(54.28$ cases per $100,000)$ is lower than that of women in the United States (101.17 cases per 100,000); however, the age adjusted mortality rate is higher (32 vs 28 per 100,000). Chamorros are the most populous ethnic group on Guam, making up $37 \%$ of the total population of 154,805 (US Census Bureau, 2000). Other major ethnic groups on the island include Filipinos
(26\%), Asians (13\%), non-Latino Whites $(6.8 \%)$, and Micronesians other than Chamorro $(8 \%)$.

Until recently, little was known about the breast cancer control needs of Chamorros. However during the past few years, studies conducted by National Cancer Institute funded community cancer networks (Hubbell et al., 2006 and Tanjasiri \& Tran, 2008) have begun to address this issue. For example, Balajadia et al. (2008) found that Chamorro women on Guam were relatively knowledgeable about the risk factors for breast cancer and that a high proportion $(83 \%)$ of the women over the age of 50 years 
reported receiving a mammogram within the last two years. Cruz et al. (2008) reported similar findings among Chamorro women in Southern California.

An important neglected area of research concerns the use of social support by Chamorro breast cancer survivors, the focus of the current study. Social support has frequently been associated with better emotional and physical outcomes among cancer patients in general and in breast cancer patients specifically (Burns, Craft, \& Roder, 2005 and Abercrombie, GieseDavis, Septhon, Epel, Turner-Cobb, \& Spiegel, 2004). Although some investigators report conflicting findings (Bolger, Foster, Vinokur, \& Ng, 1996 and Ferrel, Dow, \& Grant, 1995), others conclude that social support is either directly (Bloom, Stewart, Chang, \& Banks, 2004) or indirectly through coping or other constructs (Northouse, Mood, Kershaw, Schanfenacker, Mellon, Walker, et al., 2002) related to the mental health and quality of life of breast cancer survivors. The general areas of support include emotional, tangible, and informational support. Foa and Foa (1980) have recognized demonstrations of affection, status, information, money, goods and services as specific kinds of assistance to members in one's support network.

Unlike the mainland United States where there are thousands of breast cancer support groups supported by organizations such as the Komen Foundation and the American Cancer Society, few formal breast cancer survivor support programs exist on Guam. There is one breast cancer survivor support group, "Cadena de Amour Breast Cancer." In addition, the American Cancer Society sponsors "Reach to Recovery" and "Look Good: Feel Better" programs. In the Reach to Recovery Program, volunteers who are breast cancer survivors are trained to provide breast cancer information, support, and hope to other survivors. The program also provides a gift bag containing helpful pamphlets with suggestions for postoperative care, information for family members, and a temporary prosthesis. The Look Good: Feel Better Program is a free community service dedicated to helping women and men manage the appearance related side effects of chemotherapy and radiation therapy. Despite the existence of these programs, few Chamorros participate in the activities (Iyechad, 2001).

In light of the relatively limited number of formal support networks, the current study assessed the types of social support used by breast cancer survivors on Guam. This information is a critical initial step in identifying potential gaps in services and to building resources related to support for breast cancer survivors in this indigenous group of women.

\section{METHODS}

We conducted English language face-to-face interviews regarding social support among Chamorro breast cancer survivors on Guam from May 2004 to March 2005. In this study, social support refers to the information, encouragement, and tangible assistance offered to a person by others and is perceived by the person as being beneficial to her functioning. The interviews led to the construction of genograms and ecomaps that provided pictorial displays of the women's family relationships, medical history, and social networks. Genograms and ecomaps are often used by family therapists to show the many systems that may be at play in an individual's life. The University of California, Irvine Human Subjects Review Committee approved the study protocol.

\section{Research Design}

A non-probability purposive sample design was used to recruit the participants (Day, 1989). The investigators made presentations to the island's breast cancer support group, "Cadena de Amour Breast Cancer," and members of the Pacific Association for Radiation Survivors and asked for volunteers. They also partnered with three medical facilities: the U.S. Naval Hospital, Guam Memorial Hospital, and Guam Cancer Clinic to recruit participants. Finally, they collaborated with individual physicians serving as co-signers of a letter of invitation to potential participants.

\section{Sample}

To be eligible, volunteers had to be a selfidentified Chamorro woman aged 40 or older 
with a diagnosis with breast cancer. Women undergoing active treatment were excluded. We selected the first 25 women who met these criteria, taking approximately eight months of recruitment.

\section{Data Collection}

The data collection methods included conducting interviews and developing genograms and ecomaps. The investigators met individually with each of the 25 participants in locations where they felt comfortable such as the participant's home, workplace, and counseling session rooms of a private behavioral health clinic. At the completion of the interview, participants were given a gift of $\$ 25$ as a token of appreciation.

\section{Figure 1}

\section{Genogram}
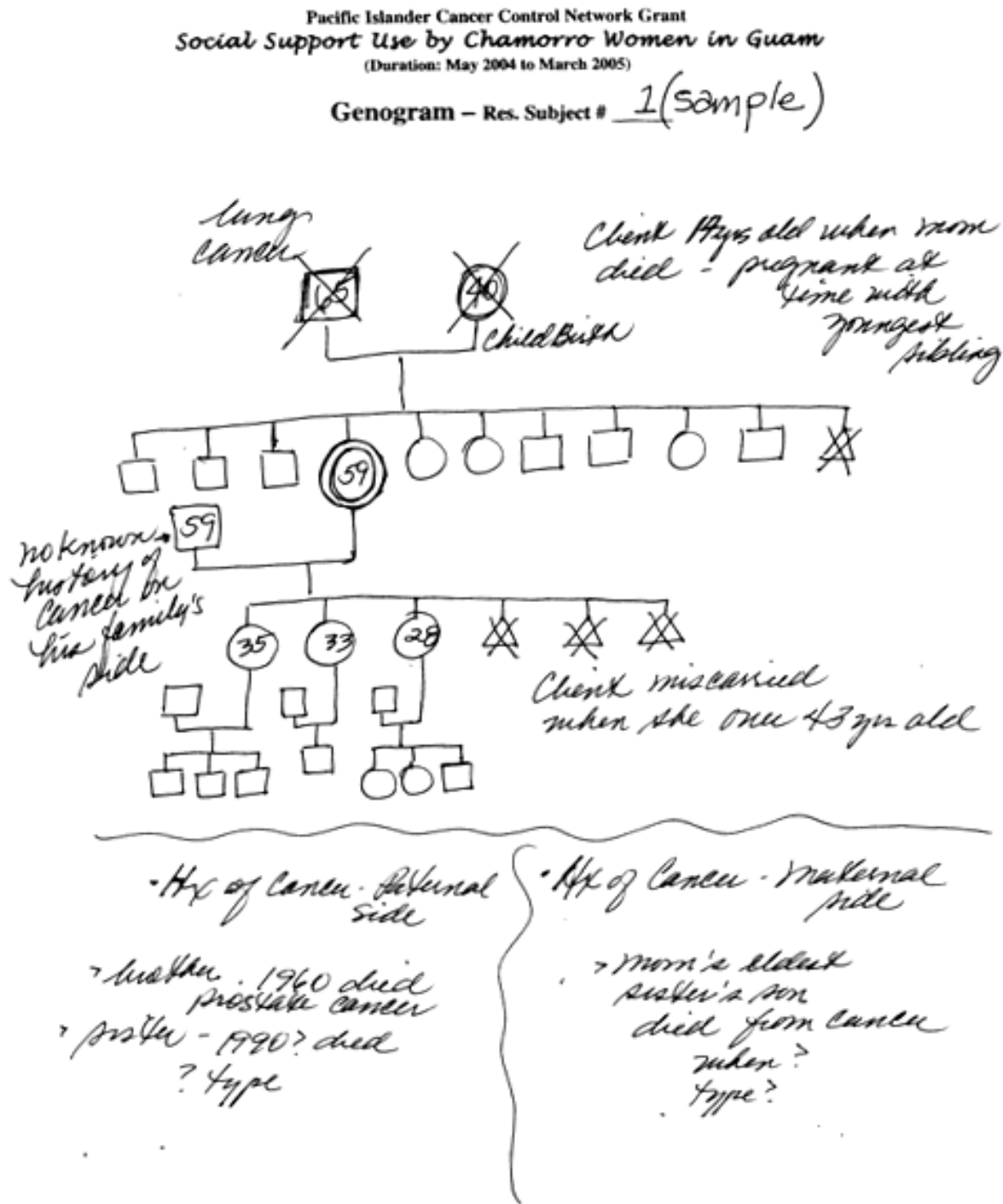

A genogram is a pictorial display of a person's family relationships and medical history. It goes beyond a traditional family tree by allowing the user to visualize hereditary patterns and psychological factors that punctuate relationships. It can be used to identify repetitive patterns of behavior and to recognize hereditary tendencies 


\section{Figure 2}

\section{Ecopmap}

Pacific Islander Cancer Control Network Grant

- Social Support Use by Chamorro Women in Guam (Duration: May 2004 to March 2005)

List of Resources,

Ecomap - Res. Subject \# 1 (sanple)

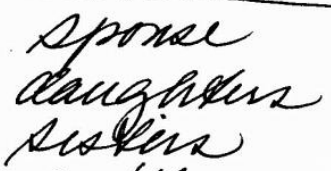

lusthers

ixllawe (fiom ofprise)

pavishiones (inayes)

\& 6 mustla man pervice

H.S. Haval Stoppital (Gn) Guam incelcel tefescal

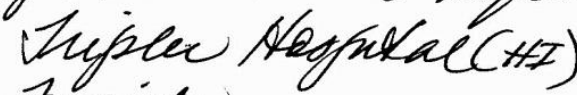

Hnende

Ist Laely.

Phone calle fun (GU)

fruñls family

sponse' \& friende

(cmpany \& fored)

Ameiceax Cancer

fociets (GUF HL)
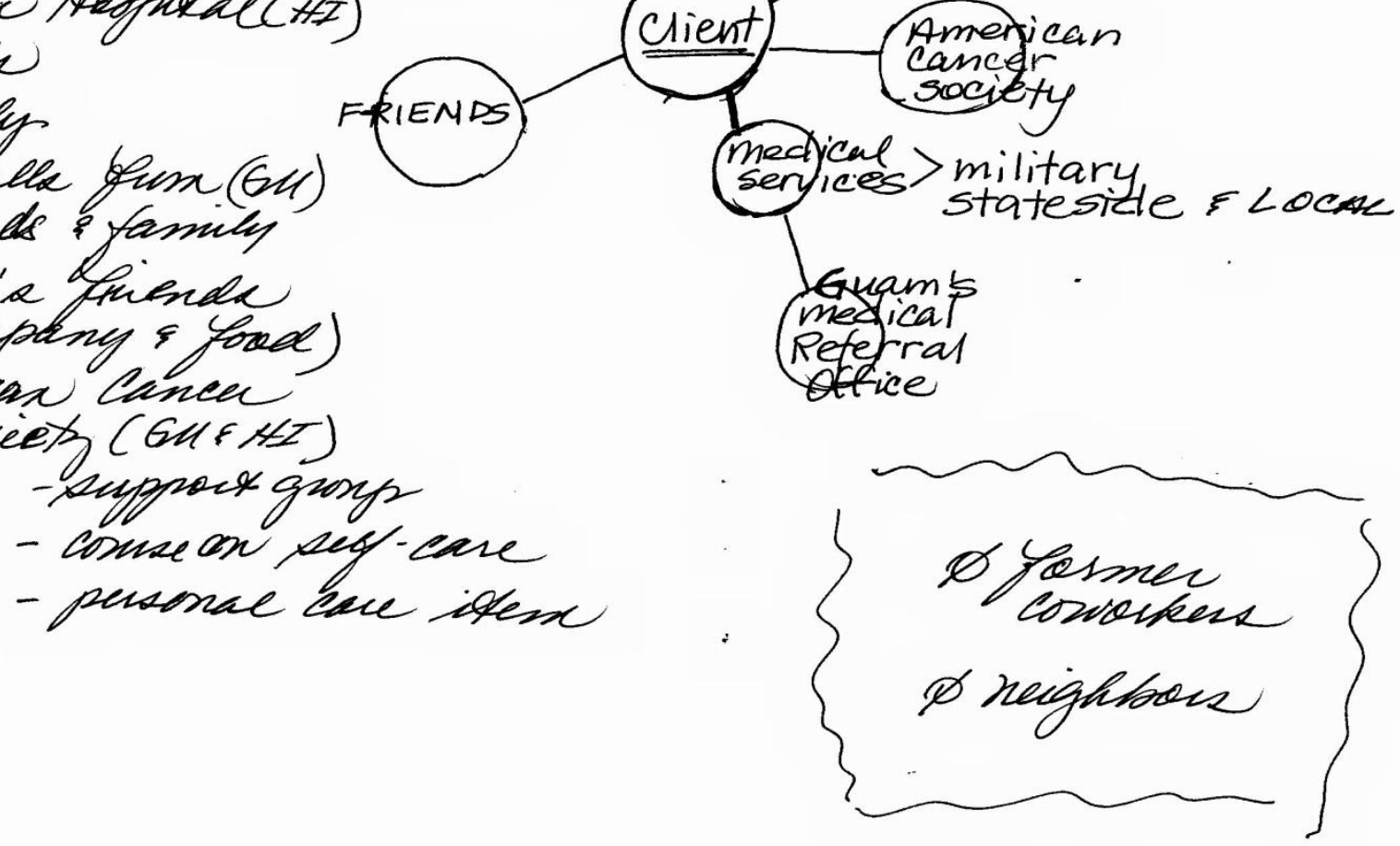

Ecomaps are visual maps of individual's or families' connection to the external world. They provide a tool for assessment of family, social and community relationships and highlight the quality of these connections. The closer the proximity to the individual, the more significant the relationship is 
The interviews began with the gathering of basic socio-demographic information. The investigators then elicited general views about the participant's experience since the diagnosis of breast cancer using a semi-structured interview guide. Next, the interviews became more focused, using systematic techniques for obtaining social support and formal network data from each participant. This data assisted researchers in determining the categories of social networks and interpersonal resources, and led to the development of genograms and ecomaps by the researchers with each participant. A genogram (Figure 1) is a pictorial display of a person's family relationships and medical history (McGoldrick \& Gerson, 1985). It goes beyond a traditional family tree by allowing the user to visualize hereditary patterns and psychological factors that punctuate relationships. It can be used to identify repetitive patterns of behavior and to recognize hereditary tendencies. The ecomap (Figure 2) is a visual assessment tool depicting the relationships between a family and its social network (Hartman, 1995). As the name signifies, research and participant together map out connections between the family and its ecological environment. Identifying these connections clarifies and organizes data on a family's environment.

In this study, we used genograms to identify all of the family relationships and cancer history related to each individual. We then used ecomaps to provide a depiction of the individual's social support system at a glance. From these constructions, the investigators ascertained the degree of influence made by different elements of a person's social support system. Examples of support systems include formal organizations, family members, and friends.

In order to compare the characteristics of our sample with those of other breast cancer survivors, we also evaluated data, including ethnicity/race, age at diagnosis, and years since diagnosis, from the Guam Cancer Registry, University of Guam.

\section{Analysis}

The analysis of the interviews was informed by McGoldrick and Gerson (1985) and Hartman (1995). The analysis began with two of the investigators (LI and $\mathrm{LN}$ ) familiarizing themselves with each of the data sets. This task required reading through the interview data and taking notes on the social networks described by each participant. Thereafter, a more formalized analysis was performed that involved creating genograms and ecomaps.

The genogram mapped the genealogical data, the family relationships, and the strength of the relationships. The genograms also highlighted family medical history relevant to cancer and initiated discussion about the manner in which the cancer was addressed within each participant family. This progressed into the creation of an ecomap outlining the resources employed by the participant during their venture with breast cancer. The ecomap provided the context of social relationships for each participant. For example, the ecomap included key people that provided support as well as those who expected similar support from the client. This included a brief description of the social relationship (family, friend colleague, or neighbor) and relevance to the participant's life. Upon completion of the ecomaps, a quantitative summary of the data was constructed by calculating the average use of the different areas of social support for participants.

\section{Results}

The mean age of the 25 participants was 54.5 years. Approximately $61 \%$ were married, and $69 \%$ were currently employed. The average number of years since the diagnosis of breast cancer was 7.8 years. Approximately $41 \%$ of the women were diagnosed within the past five years with the remainder being diagnosed more than five years prior to the study.

Some of the characteristics of the 407 known breast cancer survivors on Guam at the time of the study are shown in Table 1. Approximately 
Perez, L.A., Natividad, L., Chung, W., Haddock, R.L., Wenzel, L. \& Hubbell, F.A. / Californian Journal of Health Promotion 2010,

Volume 8, Special Issue (Cancer Control), 63 - 72

$46 \%$ of the survivors were Chamorro. The mean age at diagnosis and years since diagnosis were similar between our sample and all breast cancer survivors.

Table 1

Guam Breast Cancer Cases Alive In 2005

\begin{tabular}{cccccccccc}
\hline \hline & $\begin{array}{c}\text { Total } \\
\text { cases }\end{array}$ & Chamorro & Filipino & Micronesian & White & Asian & Black & Other $\begin{array}{c}\text { Unknown } \\
\text { ethnicity }\end{array}$ \\
\hline Total cases & 407 & 188 & 127 & 6 & 35 & 25 & 2 & 5 & 19 \\
\hline Average age at diagnosis & $54 . .97$ & 56.66 & 55.05 & 44.00 & 50.78 & 58.60 & 52.00 & 54.80 & 48.58 \\
\hline Stdev & 12.72 & 12.76 & 12.32 & 10.06 & 13.42 & 14.23 & 1.41 & 3.49 & 11.64 \\
\hline Maximum age & 89 & 90 & 90 & 57 & 83 & 82 & 53 & 61 & 66 \\
\hline Minimum age & 25 & 25 & 29 & 31 & 32 & 32 & 51 & 53 & 28 \\
\hline $\begin{array}{c}\text { Average years since } \\
\text { diagnosis }\end{array}$ & 6.099 & 6.128 & 5.409 & 4.333 & 7.543 & 4.760 & 3.000 & 11.000 & 9.842 \\
\hline Stdev & 4.72 & 4.75 & 4.39 & 5.16 & 4.15 & 3.29 & 2.83 & 3.74 & 6.91 \\
\hline Maximum years & 21 & 21 & 21 & 14 & 17 & 12 & 5 & 11 & 21 \\
\hline Minimum years & $<1$ & $<1$ & $<1$ & $<1$ & $<1$ & $<1$ & 1 & 7 & $<1$ \\
\hline \hline
\end{tabular}

Analysis of the genograms produced several themes. Multiple generations indicated that cancer was present, sometimes the same cancer type. Most cancers were traceable to the last two generations possibly due to the prior generation's limited access to medical technology. With regard to social support, the nuclear family system played a key support role. Similarly, the extended family system and nonWestern kinship (compadre system) provided ancillary support. With regard to network characteristics, the larger the family size, the greater the appearance of social support. In smaller family systems, there was a greater contribution by individual members.

Responses collected from the ecomaps were placed along a continuum that reflected those believed by participants to be innermost in comparison to those of a less intimate orientation. The respondents were most likely to list nuclear family $(n=24)$ as closest to them, or most commonly used and the most intimate social support. Several categories tied as the second-ranked resource: friends $(n=18)$, formal services $(n=18)$, and church/faith $(n=18)$. The next most frequently ranked were extended family $(n=16)$, work $(n=11)$, clubs and organizations $(n=10)$, household members $(n=2)$, neighbors $(\mathrm{n}=1)$, and the self $(\mathrm{n}=1)$.

The most common sources of support were informal with $60.2 \%$ of participants reporting contributions of this nature. The individual types of informal support included the nuclear family $(34.2 \%)$, extended family (11.5\%), friends $(8.0)$, colleagues $(3.9 \%)$, self $(1.8 \%)$, and neighbors $(0.8 \%)$. The next most common form of support was provided by church or faith identified as a resource by $21.9 \%$ of the participants. Formal 
services and various clubs and organizations were the third most commonly used support systems with $17.9 \%$ of the women citing them.

\section{Discussion}

To our knowledge, this is the first study of social support for breast cancer survivors on Guam. Our findings emphasize the importance of family as the primary social support system as reported by $45 \%$ of Chamorro breast cancer survivors, with reliance on church or faith as the next most prevalent source of support. Formal programs, such as cancer support groups and clubs, were depended upon much less commonly. The results are consistent with research that has documented the significance of these social support systems within the Chamorro culture (Iyechad, 2001). At the core of the culture is a complex social protocol centered upon respect, caring, accepting and helping one another with a strong emphasis on family. Inafa'maolek, the Chamorro word for interdependence, is a central value in the culture which depends on a spirit of cooperation. The Chamorro culture has been strongly influenced by the Spanish ever since Ferdinand Magellan first landed on Guam in the 16th century. The Catholic Church, which to this day remains the center of village activity, has probably been the most important Spanish influence. Therefore, it is not surprising that the family and church were identified as the most important sources of social support for these breast cancer survivors.

The study did not determine whether emotional or physical outcomes were improved by the types of social support used by the women. Nevertheless, the findings may provide helpful information for future planning to improve social support for Chamorro breast cancer survivors and potentially improve support for family. Because of the important role they play in providing social support, nuclear and extended family members could be included in programs designed to enhance and benefit from such services. This recommendation is supported by previous studies that assessed different types of psychosocial interventions in other populations. For example, social support interventions involving family members and psychoeducational interventions may be more effective in improving patient outcomes than those characterized by peer discussion and sharing of experiences among breast cancer survivors (Helgeson, Cohen, Schulz, \& Yasko, 2001). Psychoeducational intervention focuses on both psychological (e.g., increasing patients' mastery and feeling of control) and educational aspects (e.g., providing information about and ways of coping with the illness).

While most previous studies have been conducted with non-Latino white women, protective effects of social support for ethnic minority patients have also been shown. For example, in a study of Latina breast cancer patients, Alferi et al. (2001) found that cancer patients who perceived higher levels of emotional support from friends or instrumental support from a spouse before surgery reported lower level of distress after surgery. Similarly, Maly et al. (2005) found that non-White women with breast cancer (African Americans, Latina, and others) who received emotional support from their adult children were less likely to exhibit symptoms of depression and anxiety. Although the literature is relatively sparse with respect to our understanding of the breast cancer experience between different ethnic groups, it is worth noting that cultural influences and socioecological factors affect the survivorship experience (Ashing-Giwa et al., 2004). To that end, this study conducted among Chamorro breast cancer survivors on Guam contributes to a growing literature identifying factors which influence the breast cancer experience, in this case via family support.

Our study had important limitations. First of all, it was a small study using a non-probability purposive sample design. As such the results may not be generalizable to the larger Chamorro breast cancer survivor population. However although small, the sample contained 25 (13\%) of the 188 Chamorro breast cancer survivors and approximately $6 \%$ of all breast cancer survivors on Guam, and their characteristics were similar to other breast cancer survivors at the time of the study. Moreover, obtaining the in depth information that we desired would be very labor intensive and expensive using a larger sample. 
Thus, the methodology was appropriate for this exploratory study although the data may be subject to recall and social desirability response bias. In future studies, it would be interesting to determine support differences between nuclear and extended family and whether there were gender differences in support provision or type. In future program planning, consideration should be given to recognizing natural support resource, since strong evidence suggests that it is important to include both survivors and family in programs of care (Mellon et al., 2006). This may be especially true for Chamorro breast cancer survivors on Guam, particularly if formal support mechanisms are sparse, not accessible or unattended.

These results provide a window from which to begin to understand potential patterns of cultural factors which may influence health behaviors for breast cancer survivors and their family, which is recognized as an emerging area of survivorship research (Lim et al., 2009). When considering enhancing or increasing the number of breast cancer survivor groups or educational efforts on Guam, it will be important to incorporate these sociocultural and relational factors in program building.

\section{References}

Abercrombie, H.C., Giese-Davis, J., Sephton, S., Epel, S., Turner-Cobb, J. M., \& Spiegel, D. (2004). Flattened cortisol rhythms in metastatic breast cancer patients. Psychoneuroendocrinology, 29, 1082-1092.

Alferi, S.M., Carver, C.S., Antoni, M.H., Weiss, S., \& Duran, R.E. (2001). An exploratory study of social support, distress, and life disruption among low-income Hispanic women under treatment for early stage breast cancer. Health Psychology, 20(1), 41-6.

Ashing-Giwa, K.T., Padilla, G., Tejero, J., Kraemer, J., Wright, K., Coscarelli, A., Clayton, S., Williams, I., Hills, D. (2004). Understanding the breast cancer experience of women: a qualitative study of African American, Asian American, Latina and Caucasian cancer survivors. Psychooncology, 13(6), 408-28.

Balajadia, R.G., Wenzel, L., Huh, J., Sweningson, J., \& Hubbell, F.A. (2008). Cancer-related knowledge, attitudes, and behaviors among Chamorros on Guam. Cancer Detection and Prevention, 32S, 415.

Bloom, J.R., Stewart, S.L., Chang, S., \& Banks, P.J. (2004). Then and now: quality of life of young breast cancer survivors. Psychooncology, 13(3), 147-60.

Bolger, N., Foster, M., Vinokur, A.D., \& Ng, R. (1996). Close relationships and adjustment to a life crisis: the case of breast cancer. Journal of Personality and Social Psychology, 70(2), 283-94.

Burns, C.M., Craft, P,S., \& Roder D.M. (2005). Does emotional support influence survival? Findings from a longitudinal study of patients with advanced cancer. Support Care Cancer, 13(5), 295-302.

Cruz, A.L.H., Chung, W., Huh, J., Blas, L.A., Cruz, L.A.C., Hubbell, F.A., \& Wenzel, L. (2008). Breast cancer screening among Chamorro women in California, Cancer Detection and Prevention, 32S, $16-22$.

Day, L. (1989). Designing and conducting health surveys. San Francisco: Jossey-Bass Publishers.

Foa, E.B. \& Foa, U.G. (1980). Resource theory: interpersonal behavior as exchange. In: Gergen, K.J., Greenberg, M. S., \& Willis, R. H., (Eds). Social Exchange: Advance in Theory and Research (pp. 77-94). New York: Plenum Press.

Ferrell, B.R., Dow, K.H., \& Grant, M. (1995). Measurement of the quality of life in cancer survivors. Quality of Life Research, 4(6), 523-31.

Haddock, R.L., \& Naval, C.L (2002). Cancer on Guam, especially among Micronesians. Pacific Health Dialogue, 9(2), 222-224.

Haddock, R.L., Talon, R.J., \& Whippy, H.J.D. (2006). Ethnic disparities in cancer mortality among residents of Guam. Asian Pacific Journal of Cancer Prevention, 7, 411-414.

Hartman, A. (1995). Diagrammatic assessment of family relationships. Families in Society, 76, 111-122. 
Helgeson, V.S., Cohen, S., Schulz, R. \& Yasko, J. (2001). Group support interventions for people with cancer: benefits and hazards. In: Baum, A. \& Andersen, B.L. (eds). Psychosocial intervention for cancer (pp. 269-286). Washington: American Psychological Association.

Hubbell, F.A., Luce, P.H., Afeaki, W.P., Cruz, L.A., Mummert, A., McMullin J.M., Pouesi, J., Reyes, M.L., Taumoepeau, L.T., Tu'ufuli, G.M., \& Wenzel, L. (2006). Legacy of the Pacific Islander Cancer Control Network. Cancer, 107(S), 2091-98.

Iyechad, L. (2001). An Historical Perspective of Helping practices associated with birth, marriage, and death among Chamorros of Guam. New York: the Edwin Mellen Press.

Lim, J.W., Gonzalez, P., Wang-Letzkus, M.F., \& Ashing-Giwa, K.T. (2009). Understanding the cultural health belief model influencing health behaviors and health-related quality of life between Latina and Asian-American breast cancer survivors. Support Care Cancer, 17(9), 1137-47.

Maly, R.C., Umezawa, Y., Leake, B., \& Silliman, R.A. (2005). Mental health outcomes in older women with breast cancer: impact of perceived family support and adjustment. Psychooncology, 14(7), $535-45$.

McGoldrick, M. \& Gerson, R. (1995). Genograms in family assessment. New York: W.W. Norton.

Mellon, S., Northouse, L.L., \& Weiss, L.K. (2006). A population-based study of the quality of life of cancer survivors and their family caregivers. Cancer Nursing, 29(2), 120-31.

Northouse, L.L., Mood, D., Kershaw, T., Schafenacker, A., Mellon, S., Walker, J., et al. (2002). Quality of life of women with recurrent breast cancer and their family members. Journal of Clinical Oncology, 20(19), 4050-64.

Tanjasiri, S.P., Tran, J.H. (2008). Community capacity for cancer control collaboration: weaving an Islander Network for Cancer Awareness, Research and Training for Pacific Islanders in Southern California. Cancer Detection and Prevention, 32 (S1), S37-40.

U.S. Census Bureau, 2000 Census of Population and Housing, Social, Economic, and Housing Characteristics. PHC-4-GUAM, Guam. Washington, DC (2003). Available at http://www.census.gov/prod/cen2000/phc-4-guam.pdf. Accessed September 10, 2010.

Author Information

Lilli Ann Perez, Ph.D.

Family Pacific

Lisalinda Natividad, M.S.W.

Family Pacific

William Chung, B.S.

Health Policy Research Institute

Robert L. Haddock, D.V.M., M.P.H.

University of Guam,

Cancer Research Center of Guam, UOG Station

Lari Wenzel, Ph.D.

Health Policy Research Institute

University of California, Irvine,

Department of Medicine and Chao Family Comprehensive

Cancer Center 
Perez, L.A., Natividad, L., Chung, W., Haddock, R.L., Wenzel, L. \& Hubbell, F.A. / Californian Journal of Health Promotion 2010, Volume 8, Special Issue (Cancer Control), 63 - 72

*F. Allan Hubbell M.D., M.S.P.H.

Health Policy Research Institute

University of California, Irvine

264 Irvine Hall

1001 Health Sciences Road

Irvine, CA 92697-3950

Telephone: (949) 824-3975

Fax: (949) 824-2676

Email: fahubbel@uci.edu

* corresponding author 